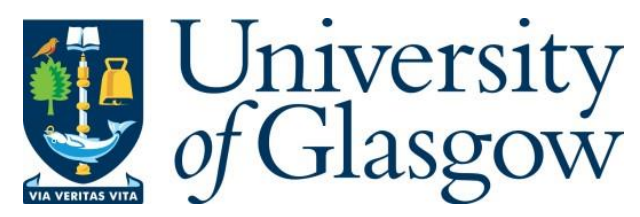

Huang, Y., Karadimas, P. and Pour Sohrab, A. (2020) Multi-Antenna Array Topologies Optimization for Future Wireless Networks by Employing Particle Swarm Optimization. In: 2019 PhotonIcs Electromagnetics Research Symposium - Spring (PIERS-Spring), Rome, Italy, 17-20 Jun 2019, pp. 4138-4143. ISBN 9781728134048.

There may be differences between this version and the published version. You are advised to consult the publisher's version if you wish to cite from it.

http://eprints.gla.ac.uk/227381/

Deposited on: 8 January 2021

Enlighten - Research publications by members of the University of Glasgow http://eprints.gla.ac.uk 


\title{
Multi-Antenna Array Topologies Optimization for Future Wireless Networks by Employing Particle Swarm Optimization
}

\author{
Y. K. Huang, P. Karadimas, A. P. Sohrab \\ School of Engineering, University of Glasgow \\ Glasgow, G12 8QQ UK
}

\begin{abstract}
The purpose of this work is to design novel antenna array topologies that maximize channel capacity. Accordingly, we employ the particle swarm optimization (PSO) algorithm as an effective technique in similar electromagnetic problems. We use a novel modelling approach to derive the spatial fading correlation function by employing a generic three-dimensional (3-D) angle of arrival (AOA) model. We then employ the PSO algorithm to find the antenna array topology that maximizes the channel capacity. The use of the PSO algorithm together with the new modelling approach for the spatial correlation is sufficiently tested and verified in terms of efficiency and correctness of addressing similar type of electromagnetic problems. Moreover, the general AOA model of this work is adaptable to different propagation environments, thus our approach is very promising in this regard.
\end{abstract}

Keywords: Antenna array topologies, angle of arrival model, channel capacity, particle swarm optimization, spatial correlation function.

\section{INTRODUCTION}

In existing and future wireless systems, antenna arrays play an important role for capacity and signal quality enhancement. Since the space limitation is one of the main constraints in antenna array synthesis, finding optimum locations of antenna elements yielding high data rates is a significant issue for antenna array design. For convenience, a widely used "rule" is that half a wavelength $0.5 \lambda$ separation is required to obtain decorrelation between antenna elements. However, it has been observed that different array configurations obtained by PSO outperform the conventional uni form linear arrays with $0.5 \lambda$ length spacing in terms of MIMO capacity [1]. Various evolutionary metaheuristic algorithms have been used to solve antenna topology problems by using different objective functions, for example minimizing sidelobe level (SLL) for high directionality and interference suppression [2], minimizing lower bound of the mean square estimation error for enhancement of direction of arrival (DOA) estimation accuracy [3] and so on. However, spatial correlation suppression and channel capacity maximization by optimizing the array element location within a limited three-dimensional (3-D) space has not been sufficiently addressed.

For capacity maximization, genetic algorithm (GA) was used in [4] to optimize channel capacity in terms of selecting the optimal number of the antenna array elements at the base station, in which a discrete model for the AOA distribution, together with different fixed array configurations were considered. The work of [5] proposed a GA to obtain both the location and orientation of each MIMO array antenna with fixed number of elements that maximized the ergodic capacity, which outperformed the standard uniform linear array (ULA) and uniform circular array (UCA) for a given propagation channel in the comparisons. Another work in [6] implemented PSO to find the optimal locations of antenna elements in a 3-D space and maximized the ergodic channel capacity with a closed-form spatial correlation function. This work has also verified the effectiveness of applying PSO in antenna array topology optimization problems. However, they only considered the uniform AOA distribution.

In this paper, we first derive and approximate spatial correlation function considering a 3-D model for Gaussian AOA distribution. The spatial correlation matrices construct the MIMO channel matrix, from which we then carry out the calculation of channel capacity by using the PSO algorithm. We find the maximal channel capacity with the help of PSO by searching the location information of antenna array elements in 3-D space.

This paper is organized as follows: Section II describes the theoretical and mathematical derivations, including antenna array geometry definition, AOA distribution, spatial correlation function, MIMO Kronecker channel model, channel capacity and PSO algorithmic process. Section III presents simulation results to verify the correctness and feasibility of the proposed method. Finally, conclusions are drawn in section IV.

\section{THEORETICAL/MATHEMATICAL DESCRIPTION}

\section{A. Array Geometry}

Let the $m$ th element of antenna array be located at the location $\left(x_{m}, y_{m}, z_{m}\right)$ in space as shown in Fig. 1. For illustration, there are four antenna elements with corresponding locations in the 3-D spatial coordinate $(\mathrm{x}, \mathrm{y}, \mathrm{z})$ system. They form a receiver antenna array topology with origin as the phase reference point. The directional impinging signal involves both elevation AOA $\theta$ and azimuth AOA $\varphi$. Therefore, the wave vector of a single multipath component with AOA $(\theta, \varphi)$ in the 3-D space of Fig. 1 can be expressed by [6] 


$$
\mathbf{k}(\theta, \varphi)=\frac{2 \pi}{\lambda}\left[\begin{array}{c}
\sin (\theta) \cos (\varphi) \\
\sin (\theta) \sin (\varphi) \\
\cos (\theta)
\end{array}\right]
$$

Let $j=\sqrt{-1}, \mathbf{r}_{m}=\left(\begin{array}{lll}\mathbf{x}_{m} & \mathbf{y}_{m} & \mathbf{z}_{m}\end{array}\right)^{T}$ indicate the location vector of the $m$ th antenna element in the 3-D space (see Fig. 1), where the superscript " $T$ " represents the transpose operation. Accordingly, the phase delay of the steering vector of a multipath component with direction angle $(\theta, \varphi)$ impinging on the $m$ th array element can be expressed by [6]

$$
\begin{aligned}
& v_{m}(\theta, \varphi)=\exp \left\{-j\left[\mathbf{r}_{m} \cdot \mathbf{k}(\theta, \varphi)\right]\right\}= \\
& \exp \left\{-j(2 \pi / \lambda)\left[x_{m} \sin (\theta) \cos (\varphi)+\right.\right. \\
& \left.\left.y_{m} \sin (\theta) \sin (\varphi)+z_{m} \cos (\theta)\right]\right\} .
\end{aligned}
$$

Due to the spatial relation between the spherical coordinate and the rectangular coordinate, the difference vector between $\mathbf{r}_{m}$ and $\mathbf{r}_{n}$ can be similarly expressed as

$$
\mathbf{d}_{m, n}=\mathbf{r}_{m}-\mathbf{r}_{n}=\left[\begin{array}{c}
r_{m, n} \sin \left(\beta_{m, n}\right) \cos \left(\alpha_{m, n}\right) \\
r_{m, n} \sin \left(\beta_{m, n}\right) \sin \left(\alpha_{m, n}\right) \\
r_{m, n} \cos \left(\beta_{m, n}\right)
\end{array}\right],
$$

in which $r_{m, n}=\sqrt{\left(x_{m}-x_{n}\right)^{2}+\left(y_{m}-y_{n}\right)^{2}+\left(z_{m}-z_{n}\right)^{2}}$, $\beta_{m, n}$ and $\alpha_{m, n}$ denote the angle between $\mathbf{d}_{m, n}$ and the $\mathrm{Z}$ axis and the angle between $\mathbf{d}_{m, n}$ and the $\mathrm{X}$ axis, respectively.

\section{B. Angle of Arrival}

The signal arriving at the received antenna array can be described by the incident wave with the AOA $(\theta, \varphi)$. The existing correlation models are derived for a particular AOA distribution such as uniform, Gaussian, Von Mises, cosine, Laplacian and so on [7]. This paper concentrates the analysis on the Gaussian AOA distribution. The angular spread is defined as the standard deviation of the AOA distribution [8].

Assume that the azimuth and elevation AOA are independent and both follow Gaussian distribution, the joint AOA distribution can be defined as [8]

$$
\begin{aligned}
P(\theta, \varphi)=\frac{C_{\theta}}{\sqrt{2 \pi} \sigma_{\theta}} \exp \left[-\frac{\left(\theta-\theta_{0}\right)^{2}}{2 \sigma_{\theta}{ }^{2}}\right] \times & \\
& \frac{C_{\varphi}}{\sqrt{2 \pi} \sigma_{\varphi}} \exp \left[-\frac{\left(\varphi-\varphi_{0}\right)^{2}}{2 \sigma_{\varphi}{ }^{2}}\right],
\end{aligned}
$$

where $\theta \in[0, \pi]$ and $\varphi \in[-\pi, \pi]$ as illustrated in Fig. 1, and $\sigma_{\theta}, \sigma_{\varphi}$ are the standard deviations in the elevation and azimuth plane, respectively. $\theta_{0}$ and $\varphi_{0}$ denote mean elevation AOA (MEOA) and mean azimuth AOA (MAOA). Moreover, $C_{\theta}$ and $C_{\varphi}$ are the normalization factors to guarantee that $P(\theta, \varphi)$ is a probability density function.

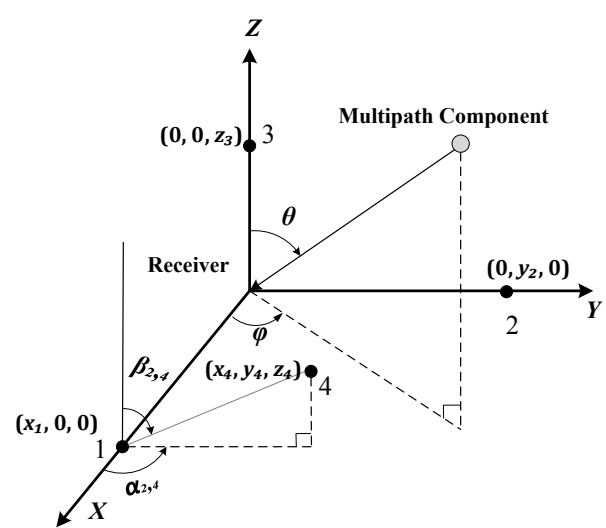

Figure 1. A 3-D coordinate system with 4 array elements in space.

\section{Spatial Correlation}

The spatial correlation between the $m$ th and the $n$th array elements in space is given by [6]

$$
\begin{aligned}
R_{s}(m, n)= & E\left\{v_{m}(\theta, \varphi) v_{n}(\theta, \varphi)^{*}\right\}= \\
& \int_{\theta} \int_{\varphi} v_{m}(\theta, \varphi) v_{n}(\theta, \varphi)^{*} P(\theta, \varphi) \sin (\theta) d \varphi d \theta
\end{aligned}
$$

where $(.)^{*}$ denotes the conjugate transpose.

Using eqs. (1) - (4), we can expand the spatial correlation of (5) as

$R_{s}(m, n)=$

$\int_{\theta} \int_{\varphi} \frac{C_{\theta} C_{\varphi}}{2 \pi \sigma_{\theta} \sigma_{\varphi}} \exp \left[-\frac{\left(\theta-\theta_{0}\right)^{2}}{2 \sigma_{\theta}^{2}}\right] \exp \left[-\frac{\left(\varphi-\varphi_{0}\right)^{2}}{2 \sigma_{\varphi}^{2}}\right] \times$

$\exp \left\{j 2 \pi r_{m, n}\left[\sin \left(\beta_{m, n}\right) \sin (\theta) \cos \left(\alpha_{m, n}-\varphi\right)+\right.\right.$

$$
\left.\left.\cos \left(\beta_{m, n}\right) \cos (\theta)\right]\right\} \sin (\theta) d \varphi d \theta
$$

To avoid complicated numerical integration, we resort to Gauss-Hermite Quadrature (GHQ) to approximate the spatial correlation in (6) [8]. Due to space limitations, the proof is omitted. The approximation is as follows

$$
\begin{aligned}
& R_{s}(m, n) \approx \frac{C_{\theta}}{\sqrt{\pi}} \cdot \frac{C_{\varphi}}{\sqrt{\pi}} \times \\
& \sum_{q=1}^{M}\left\{\omega_{q} \cdot \exp \left[j \frac{2 \pi}{\lambda} r_{m, n} \cos \left(\beta_{m, n}\right) \times \cos \left(\sqrt{2} \Delta \theta \cdot x_{q}+\theta_{0}\right)\right] \times\right. \\
& \sin \left(\sqrt{2} \Delta \theta \cdot x_{q}+\theta_{0}\right) \times \sum_{p=1}^{N}\left(\omega _ { p } \cdot \operatorname { e x p } \left[j 2 \pi r_{m, n} \sin \left(\beta_{m, n}\right) \times\right.\right. \\
& \left.\left.\left.\quad \sin \left(\sqrt{2} \Delta \theta \cdot x_{q}+\theta_{0}\right) \times \cos \left(\alpha_{m, n}-\sqrt{2} \Delta \varphi \cdot x_{p}-\varphi_{0}\right)\right]\right)\right\},
\end{aligned}
$$

where the elevation angular spread (ES) $\Delta \theta$ and azimuth angular spread (AS) $\Delta \varphi$ refer to the standard deviations $\sigma_{\theta}, \sigma_{\varphi}$ respectively. We employ ES and AS from now on to denote the standard deviations. The $x_{q}(q=1,2, \ldots M), x_{p}(p=1,2, \ldots, N)$ are the roots of corresponding $M$-th and $N$-th order Hermite polynomials, and $\omega_{q}, \omega_{p}$ are the associated weights given as 


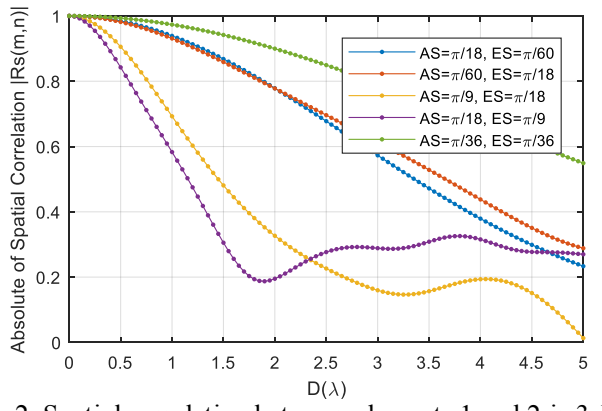

Figure 2. Spatial correlation between elements 1 and 2 in 3-D space with $\mathrm{MEOA}=\pi / 9$ and MAOA $=-\pi / 4$.

$$
\begin{aligned}
& \omega_{q}=\frac{2^{q-1} q ! \sqrt{\pi}}{q^{2}\left[H_{q-1}\left(x_{q}\right)\right]^{2}}, \\
& \omega_{p}=\frac{2^{p-1} p ! \sqrt{\pi}}{q^{2}\left[H_{p-1}\left(x_{p}\right)\right]^{2}} .
\end{aligned}
$$

\section{Kronecker Channel Model and Capacity}

Kronecker model not only allows for independent antenna array optimization at both transmitter and receiver, but also simplifies the analytical simulation and calculation. Assume a MIMO communication system with a transmit array of $N_{t}$ antennas and a receive array of $N_{r}$ antennas. We can express the $N_{t} \times N_{r}$ channel matrix under the Rayleigh fading channel $\mathbf{H}$ as follows [6]

$$
\mathbf{H}=\left(\mathbf{R}_{r x}\right)^{1 / 2} \mathbf{H}_{\omega}\left\{\left(\mathbf{R}_{t x}\right)^{1 / 2}\right\}^{H},
$$

where the superscript " $\mathrm{H}$ " denotes the complex conjugate transpose, and $\mathbf{H}_{\omega}$ is a stochastic $N_{t} \times N_{r}$ matrix with i.i.d. complex Gaussian entries with zero mean and unit variance. The $N_{r} \times N_{r}$ and $N_{t} \times N_{t}$ matrices $\mathbf{R}_{r x}$ and $\mathbf{R}_{t x}$ are the spatial correlations of MIMO antenna arrays at the receiver and transmitter sides, respectively. Thus, the corresponding full channel correlation matrix $\mathbf{R}$ (in which $\otimes$ denotes the Kronecker product) is derived as

$$
\mathbf{R}=\mathbf{R}_{r x} \otimes \mathbf{R}_{t x} .
$$

In information theory, capacity means the highest data rate to transmit reliable information over a communication channel, and it decides the success or failure of wireless communication [9]. Assume that the channel state information is completely known at the receiver while the transmitter has no channel state information, thus, the MIMO ergodic channel capacity can be expressed by [10]

$$
C=\log _{2}\left\{\operatorname{det}\left(\mathbf{I}+\rho \mathbf{H}^{H} \mathbf{H}\right)\right\},
$$

where det denote the determinant, $\mathbf{I}$ is an $N_{t} \times N_{r}(t=r)$ identity matrix, and the average signal-to-noise ratio (SNR) per receive antenna $\rho$ is set to $\rho=\frac{P}{N_{t} \sigma_{n}^{2}}$, in which $\mathrm{P}$ denotes the total power transmitted at the transmitter and $\sigma_{n}^{2}$ the noise variance. In this paper, the channel

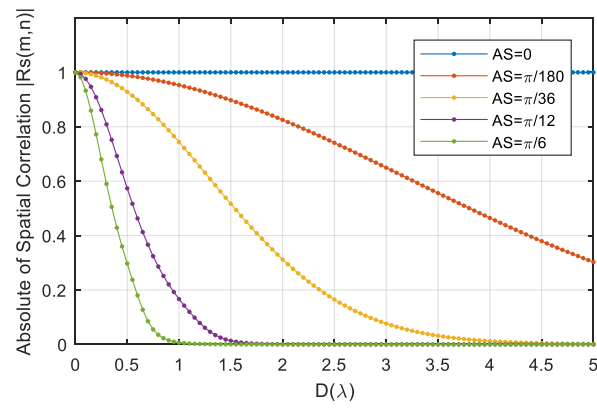

Figure 3. Spatial correlation between elements 1 and 2 in 2-D space with $\mathrm{MAOA}=\pi / 6$

capacity defined above is used as the objective function for PSO processing to be maximized.

\section{E. Particle Swarm Optimization}

PSO is widely deployed in various fields taking advantage of a swarm with multiple particles to solve multidimensional optimization problems. PSO uses the information of social interaction between independent agents and fitness to find the optimal solution

The formulas of velocity $\mathbf{v}_{i}$ and position $\mathbf{x}_{i}$ update for the $i$ th particle at each moment $t(t=0,1,2, \ldots)$ are given by [11]

$$
\begin{gathered}
\mathbf{v}_{i}(t+1)= \\
\omega \mathbf{v}_{i}(t)+c_{1} r_{1}\left[\mathbf{p}_{i}(t)-\mathbf{x}_{i}(t)\right]+c_{2} r_{2}\left[g(t)-\mathbf{x}_{i}(t)\right] \\
\mathbf{x}_{i}(t+1)=\mathbf{x}_{i}(t)+\mathbf{v}_{i}(t+1)
\end{gathered}
$$

where $\mathbf{p}_{i}(t)$ and $g(t)$ are personal best (pbest) position and global best (gbest) position respectively. $\omega \sim(0,1)$ denotes inertial weight which keeps the particle not to be affected by the pull of gbest and pbest. $c_{1}$ and $c_{2}$ are scaling factors namely acceleration coefficients for pbest and gbest respectively. $r_{1}$ and $r_{2}$ are random variables between $(0,1)$.

\section{Simulation Results}

Firstly, we observe the relation between spatial correlation and antenna spacing under our spatial correlation model. Setting two antenna array elements 1 and 2 located at $(-\sqrt{2} D / 3,-\sqrt{2 / 3} D,-D / 3)$ and $(0,0, D)$ in the space in Fig. 1, where $\mathrm{D}$ (in $\lambda)$ is a scalar defining the spacing between two elements. Assume that the $M E O A=\pi / 9$ and $M A O A=-\pi / 4$. We employ $\mathrm{M}=\mathrm{N}=5$ for the summation terms of Hermite polynomials in (7). Fig. 2 shows the absolute values of spatial correlation function between elements 1 and 2 versus D for different combinations of AS and ES. It is apparent from Fig. 2 that increasing antenna spacing between elements reduces their correlation generally. We can also observe that changing either the AS and ES has impact on spatial correlation, the spatial correlation decreases more quickly as angular spread increases.

Secondly, we remove the elevation component in (7) to obtain a 2-D spatial correlation model, which can be easily done by setting $\beta_{m, n}=\pi / 2, \mathrm{MEOA}=0$ and $\mathrm{ES}=0 . \quad$ We still select the elements 1 $(-\sqrt{2} D / 3,-\sqrt{2 / 3} D,-D / 3)$ and $2(0,0, D)$. The 


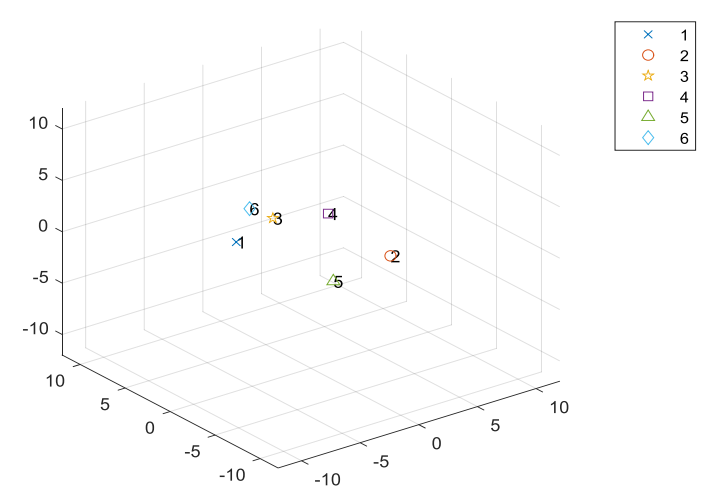

Figure 4. Six antenna array elements with optimal locations and maximal channel capacity in 3-D space.

parameters are set as: $M A O A=\pi / 6, \mathrm{M}=\mathrm{N}=5$. Fig. 3 depicts the absolute values of spatial correlation functions between elements 1 and 2 versus D for different AS under 2-D model. It is clear to see that the spatial correlation curve decreases more rapidly as the AS increases similarly.

Finally, we execute the proposed method presented in section II for channel capacity maximization. We employ PSO to find the optimal topology for six antenna array elements in 3-D space under the Gaussian AOA distribution. The parameters used are as follow: $N_{t}=N_{r}=6, M E O A=\pi / 9, M A O A=\pi / 2, E S=\pi / 36$, $A S=\pi / 60$. The boundary radius of the searching space is $D_{\max }=10 \lambda$, the spatial correlation matrix for transmitter antenna array $\mathbf{R}_{t x}$ is set to be an identity matrix and $\rho=1$. The summation terms for Hermite polynomials are $\mathrm{M}=\mathrm{N}=5$. For the algorithm, we choose 16 particles running for 500 times in the limited space. The antenna array topology after capacity optimization is shown in in Fig. 4. Under the above assumptions, the achieved channel capacity is $11.5428 \mathrm{bits} / \mathrm{s} / \mathrm{Hz}$. For comparison, we use the same conditions above for the ULA with spacing $2 D_{\max }=20 \lambda$. The resulting capacity for ULA is $5.8615 \mathrm{bits} / \mathrm{s} / \mathrm{Hz}$, which is significantly lower than the capacity obtained by our proposed modelling approach.

\section{CONCLUSION}

In this paper, a closed-form expression based on GaussHermite approximation for the spatial correlation function has been derived with respect to antenna array element locations, MAOA, MEOA, AS and ES with Gaussian AOA distribution. The results were obtained by finding the topology at the receiver side via the use of Kronecker model. Similarly, the proposed spatial correlation and optimization methodology can be used at the transmitter side. It has been observed from the simulation results that the spatial correlation between two antenna array elements versus the spacing decreases more rapidly as the angular spread increases. Based on the proposed spatial correlation model, we have employed PSO algorithm to find the optimal antenna array topology in 3-D space for channel capacity maximization. It was verified that the obtained antenna array topology with maximal channel capacity considerably outperforms ULA under the same conditions. Moreover, the generalized and flexible modeling approach proposed in this work can be used for arbitrary number of antenna elements and adapt to any realistic propagation environment.

\section{ACKNOWLEDGMENT}

This work was supported by the Engineering and Physical Sciences Research Council (EPSRC) under grant EP/R027641/1: Bandwidth and Energy Efficient Compact Multi-Antenna Systems for Connected Autonomous Vehicles.

\section{REFERENCES}

[1] U. Olgun, C. A. Tunc, D. Aktas, V. B. Erturk, and A. Altintas, "Optimization of linear wire antenna arrays to increase MIMO capacity using swarm intelligence," in The Second European Conference on Antennas and Propagation, 2007, no. 2, pp. 340340.

[2] D. Mandal, S. P. Ghoshal, and A. K. Bhattacharjee, "Application of evolutionary optimization techniques for finding the optimal set of concentric circular antenna array," Expert Systems with Applications, vol. 38, no. 4, pp. 2942-2950, 2011.

[3] P. D. Karamalis, A. G. Kanatas, and P. Constantinou, "A genetic algorithm applied for optimization of antenna arrays used in mobile radio channel characterization devices," IEEE TRANSACTIONS ON INSTRUMENTATION AND MEASUREMENT, vol. 58, no. 8 , pp. $2475-2487,2009$

[4] M. A. Mangoud, "Optimization of channel capacity for in-door MIMO systems using genetic algorithm," Progress In Electromagnetics Research C, vol. 7, pp. 137-150, 2009.

[5] M. O. Binelo, A. L. F. de Almeida, and F. R. P. Cavalcanti, "MIMO array capacity optimization using a genetic algorithm,' IEEE Transactions on Vehicular Technology, vol. 60, no. 6, pp. 2471-2481, 2011.

[6] J.-H. Lee and C.-C. Cheng, "Spatial correlation of multiple antenna arrays in wireless communication systems," Progress In Electromagnetics Research, vol. 132, pp. 347-368, 2012.

[7] Q.-U.-A. Nadeem, A. Kammoun, M. Debbah, and M. Alouini, "A Generalized spatial correlation model for 3D MIMO channels based on the Fourier coefficients of power spectrums," IEEE Transactions on Signal Processing, vol. PP, no. 99, pp. 1-1, 2015.

[8] L. Zhang, Z. Luo, and S. H. Leung, "An efficient approximation of spatial correlation based on Gauss-Hermite quadrature," IEEE Transactions on Signal Processing, vol. 66, no. 3, pp. 617-626, 2018.

[9] G. D. Durgin, "Space-time wireless channels." Prentice Hall Professional, 2003.

[10] M. K. Çolakoglu and M. Safak, "On the MIMO channel capacity predicted by Kronecker and Müller models," Wireless Personal Communications, vol. 47(1), pp. 91-100, 2008.

[11] J. Robinson and Y. Rahmat-Samii, "Particle swarm optimization in electromagnetics," IEEE Transactions on Antennas and Propagation, vol. 52, no. 2, pp. 397-407, 2004. 